\title{
Review of: "Genome-wide association analysis of cotton salt stress response-related sites"
}

\author{
NUSRAT J. KASI
}

Potential competing interests: The author(s) declared that no potential competing interests exist.

This manuscript aims to identify Genome-wide association analysis of cotton salt stress response-related sites. Meanwhile, I personally think that the topic content of this MS is also suitable for the international audiences. While, there are a number of issues that need to be addressed sufficiently.

1. Need to rewrite abstract because of repetition, same paragraph has written as introduction and number of traits or detail about traits explained after results, it should explain before results as well as results are not clear in abstract so add some more detail.

2. Set Table:1 format and add some more detail about samples like their origin, accession numbers etc.

3. In introduction part every paragraph should link with each other but here there is no any connection between them.

4. Add some information about other countries those are facing salinization problem and severity of salt stress in the form of table in introduction part.

5. Methodology is not clear. Remove headings from methodology portion because it is research paper not a review paper. Compress this part but choose those relevant and meaning full words those clear the concept.

6. Explain table 2. What it is?

7. Add phylogenetic tree picture.

8. Figure 4 explain what...? Need explanation.

9. Figure 5. Every picture needs explanation.

10. Explain figure 8.

11. Enlist the SNPs that was significantly associated with salt tolerance traits and salt tolerance index. 